Review

\title{
Procalcitonin and community-acquired pneumonia (CAP) in children
}

\author{
Bivona Giulia ${ }^{a}$, Agnello Luisa ${ }^{a}$, Scazzone Concetta ${ }^{a}$, Lo Sasso Bruna ${ }^{a}$, Bellia Chiara ${ }^{a}$, Ciaccio Marcello ${ }^{\text {a,b,* }}$ \\ a Sezione Biochimica Clinica e Medicina Molecolare, Dipartimento di Biopatologia e Biotecnologie Mediche, Università degli studi di Palermo, Italy \\ b UOC Medicina di Laboratorio-CoreLab, AOUP Policlinico P. Giaccone, Palermo, Italy
}

\section{A R T I C L E I N F O}

\section{Article history:}

Received 3 July 2015

Received in revised form 25 September 2015

Accepted 30 September 2015

Available online 3 October 2015

\section{Keywords:}

Procalcitonin

PCT

Pediatric CAP

Biomarkers

Children

Community-acquired pneumonia

\begin{abstract}
A B S T R A C T
The role of procalcitonin (PCT) as a biomarker for sepsis in adults is well documented, while its role in infections affecting neonatal children remains controversial. Among these infections, Community-Acquired pneumonia (CAP) has been studied extensively, because it's the second cause of death in children in developing countries, and one of the most frequent causes of hospitalization in industrialized countries.

The PubMed database and the Cochrane Library were used to search for the following keywords: CAP, procalcitonin, and children. Thirteen articles were studied to determine the role of PCT in CAP management, specifically its usefulness for distinguishing pneumococcal infections from viral and unknown infections, for predicting severity and the correct antibiotic treatment. This paper focuses on the studies performed to identify the best inflammatory biomarker for CAP management.

Although there is an increase in studies confirming the usefulness of PCT in CAP management in children, further studies are needed to have better understanding of its role for pediatric CAP management.
\end{abstract}

(C) 2015 Elsevier B.V. All rights reserved.

\section{Contents}

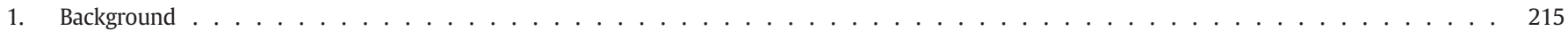

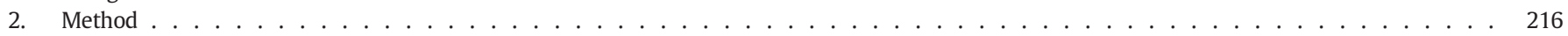

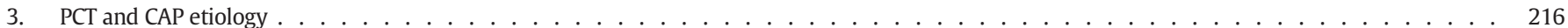

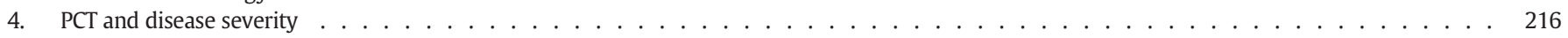

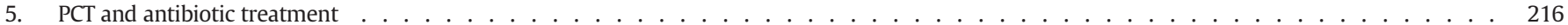

6. PCT vs other inflammatory markers . . . . . . . . . . . . . . . . . . . . . . . . . . . 217

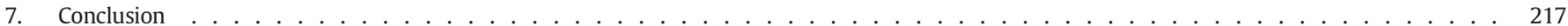

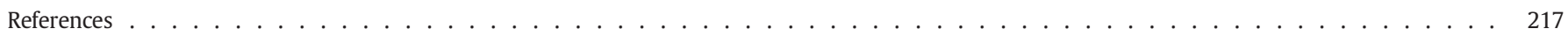

\section{Background}

The physiological role of procalcitonin (PCT) remains unknown. PCT, a protein containing 116 amino acids, is normally produced by neuroendocrine cells in the thyroid and lungs at a very low rate and it is undetectable in serum [1]. Inflammatory and infectious injuries stimulate overexpression of the CALC1 gene consequently increasing serum PCT.

Abbreviations: PCT, procalcitonin; CAP, community-acquired pneumonia; CRP, Creactive protein; ESR, erythrocyte sedimentation rate; WBC, white blood cells; ROC, receiving operating characteristic; $\mathrm{AUC}$, area under the curve; $\mathrm{CI}$, confidence interval; OR, odds ratio.

* Corresponding author at: Dipartimento di Biopatologia e Biotecnologie Mediche, Università degli Studi di Palermo, Via del Vespro, 129, 90127 Palermo, Italy.

E-mail address: marcello.ciaccio@unipa.it (C. Marcello).
Under these pathologic conditions, synthesis and secretion of PCT become ubiquitous [2].

The role of procalcitonin (PCT) as a biomarker for sepsis in adults is well documented, while its role in infections affecting neonatal children remains controversial [3]. Among these infections, Community-Acquired pneumonia (CAP) has been studied extensively, because it's the second cause of death in children in developing countries, and one of the most frequent causes of hospitalization in industrialized countries [4]. Although the significance of serum PCT levels at diagnosis is observed in adult systemic inflammation, its relevance in pediatric CAP is still uncertain [5]. The main objective of several clinicians and investigators in this field has been to detect the cause of pneumonia: since bacterial Pneumonia cannot be completely differentiated on the basis of clinical or chest radiograph findings, many efforts have been made with the goal to improve early detection of etiologic agents via biomarkers, particularly 
referring to those molecules involved in the inflammatory process. Successively, it was important to focus on the role of PCT for assessing the severity of the disease and for managing the antibiotic treatment.

This study focuses on the role of PCT in pediatric CAP management.

\section{Method}

The PubMed database and the Cochrane Library were used to search for the following keywords: CAP, procalcitonin, and children. Forty-four hits were obtained from PubMed: 30 papers and 1 review that investigate the role of PCT in adults instead of children were excluded; the same search was performed in the Cochrane Library and 2 articles, already taken from PubMed, were found. The 13 remaining articles included 4 observational studies, 3 prospective studies, 1 retrospective single center cohort study, 1 prospective single center randomized study, 1 prospective control case study, 1 prospective cohort study, 1 prospective and descriptive study and 1 review. An additional 7 articles, 1 review and 2 clinical practice Guidelines were specifically searched for as references for this study's "background", "PCT and disease severity" and "PCT and antibiotic treatment" sections. We limited the search to studies published in English, and that were conducted using clinical case studies.

\section{PCT and CAP etiology}

Several authors [6-14] have investigated the role of PCT as a serum marker for differentiating bacterial and viral CAP in children, individually or combined with other inflammatory markers. Generally, two hypotheses emerged: one confirming the usefulness of PCT in distinguishing the etiology of CAP, the other finding no usefulness.

In 2000, the study by Toikka et al. [6] showed that patients with bacterial pneumonia had a significantly higher PCT (median $2.09 \mathrm{ng} / \mathrm{ml}$ vs $0.56 \mathrm{ng} / \mathrm{ml}, \mathrm{p}=0.019$ ) and a CRP (C-reactive protein) concentration (96 mg/l vs $54 \mathrm{mg} / \mathrm{l} \mathrm{p}=0.08$ ), in 126 children (inpatients from the ages of 1 month to 17 years). Using PCT $>2 \mathrm{ng} / \mathrm{ml}$, the sensitivity was $50 \%$ and the specificity was $>80 \%$ for bacterial pneumonia. The authors concluded that PCT and CRP values greatly overlapped.

In 2001, Moulin et al. [7] investigated the role of several markers for the rapid identification of bacterial vs viral pneumonia in 72 children (inpatients form the ages 2 months to 13 years), finding that the PCT value of $1 \mathrm{ng} / \mathrm{ml}$ differentiates between bacterial infection and viral pneumonia more effectively (AUC: 0.93; 95\% CI 0.85-0.97) than CRP (AUC: 0.84, 95\% CI 0.73-0.91), and interleukin 6 (IL-6) (AUC 0.64, 95\% CI 0.45-0.80).

The study by Korppi [8] assessed the value of the clinical features in CAP for differentiating the etiology between viral, pneumococcal and atypical infections. By comparing these characteristics in 101 children (mean age: 4 years; $27 \%$ inpatients), the three nonspecific markers of typical bacterial CAP (alveolar infiltration, elevated PCT and need of hospital care), with PCT values $\geq 1 \mathrm{ng} / \mathrm{ml}$ and age $\geq 5$, were determined to be independently predictive factors for bacterial CAP (distinction of bacterial from viral etiology of CAP: OR 4.1; CI 95\% 1.0-16.6).

In 2010, the study by Lee et al. [9] compared PCT levels and other inflammatory markers between bronchopneumonia and lobar pneumonia in 76 children (mean age: 3 years; inpatients) and investigated the test performance by plotting a Receiver Operating Characteristic curve (ROC), expressed in terms of sensitivity, specificity and likelihood ratio (LR); the areas under the curves were calculated. PCT AUC at the time of admission was $0.83,95 \% \mathrm{CI} 0.65-0.99$; the maximum LR for positive results (15.3) $(\mathrm{LR}+)$ was reached at a cut-off of $2 \mathrm{ng} / \mathrm{ml}$. Results for the other inflammatory markers are reported below in the section "PCT vs other inflammatory markers".

In 2010, the Nascimento study [10] showed that in 95 children (aged 26 days to 4.8 years; inpatients) PCT had a negative predictive value for differentiating bacteraemic infections from viral infections, atypical bacterial infections and non-bacteraemic typical bacterial infections, (respectively 95\%, 89\%, 93\%) at a threshold $>2 \mathrm{ng} / \mathrm{ml}$. In addition, PCT was useful to differentiate between bacterial and viral infection (58\%).

Recently, the Galetto-Lacour study [11] confirmed the role of PCT in differentiating the etiology of CAP, particularly showing elevated PCT and CRP values to be strongly associated with pneumococcal CAP (OR: 23, 95\% CI: 5-117; OR: 19, 95\% CI: 5-75, respectively). Specifically, the authors suggested that elevated PCT or CRP values are stronger predictors of pneumococcal CAP when associated with a positive pneumococcal urinary antigen.

In order to discuss the studies with contradicting results [12-14] to the ones previously described, it could be useful to point out the results obtained by Korppi, which initially focused on the hypotheses that excluded the role of PCT in distinguishing the infection etiology. However, at a later stage the role of PCT was proven to be useful, as seen above [11-13]. Particularly, in 2001, no difference between pneumococcal, viral, mixed and unknown CAP patient subgroups was reported in 119 children from a sample of 132 children (mean age: 3 years; inpatients). PCT values of $0.5,1.0$ and $2.0 \mathrm{ng} / \mathrm{ml}$ were tested as screening limits between pneumococcal, viral and unknown pneumonia. The highest sensitivity was $55 \%$ at the $0.5 \mathrm{ng} / \mathrm{ml}$ cut-off level. The highest specificity (88\%) was reached at $1.0 \mathrm{ng} / \mathrm{ml}$. The Likelihood Ratio (LR) for positive results was 2.6 , and for negative results 0.6 , which is irrelevant. The ROC curve showed no cut-off limit with a good sensitivity and specificity for distinguishing between pneumococcal and viral pneumonia (AUC of serum PCT for differentiating viral from bacterial infections: 0.5 ).

In the study that included 190 children (aged 0-15, 30.5\% inpatients) with CAP, Korppi [13] found no differences between pneumococcal, mycoplasma/chlamydia, viral and unknown etiology infections, in terms of PCT values. The results were similar when the single strain pneumococcal infections $(0.39 \mathrm{ng} / \mathrm{ml})$ and the mixed pneumococcal infections $(0.49 \mathrm{ng} / \mathrm{ml})$ were analyzed separately.

The research by Don et al. [14] found no association between PCT levels and etiology in 68 children ( $<5$ years: $63 \%$, $>5$ years: $37 \%$; $19 \%$ inpatients), at all cut-offs, which were the 25th, 50th, 75th and 90th percentile levels, corresponding respectively to $0.4,1.0,18$ and $35.8 \mathrm{ng} / \mathrm{ml}$.

\section{PCT and disease severity}

While the relationship between PCT and severity or prognosis has been thoroughly investigated in adults during either systemic infections or CAP [15-16], only few authors [17-19] have looked into the relationship between PCT values and severity for pediatric infectious diseases. Specifically, in the cases of bacterial infections, such as meningococcal disease and urinary tract infections, or bacterial sepsis, there is an association between high PCT levels and poor prognosis. With regard to assessing CAP severity via PCT concentrations, in 2007 Don [20] conducted a prospective study performed on 100 children (mean age: 3.7 years; $26 \%$ inpatients) with the goal to evaluate the level of PCT while assessing severity of CAP, expressed in terms of needing hospital treatment and the presence of an alveolar infiltration on chest radiograph. PCT concentration showed significant difference in inpatients compared to outpatients $(\mathrm{p}<0.0012)$. Likewise, PCT levels in alveolar CAP $(25$ th75th percentiles: $0.54-22.87)$ were significantly higher $(p<0.0003)$ than levels in interstitial CAP (25th-75th percentiles: 0.31-1.04).

\section{PCT and antibiotic treatment}

Antibiotic treatment of CAP in children remains mostly empirical because the identification of etiologic pathogen is difficult in pediatric patients. The European and American guidelines [21-22] recommend beta lactam treatment as the first choice in hospitalized children of all ages, to target Streptococcus pneumoniae. In 95\% of children with bacterial pneumococcal CAP, apyrexia is reached within $48 \mathrm{~h}$ of antibiotic treatment. Since no biological predictor of clinical response to beta lactam treatment is available, in 2012 Cohen et al. [23] performed a retrospective study on 125 CAP children (mean age: 3.1 years; inpatients) treated 
with beta lactam in monotherapy, with the goal to identify the best predictor of clinical response in terms of diagnostic performance (sensitivity, specificity, likelihood ratio and predictive value) among PCT, WBC count, neutrophil count and CRP. PCT levels were significantly higher in children with, rather than without, a response to beta lactam treatment ( $3.7 \mathrm{ng} / \mathrm{ml}, 1-9.4$, vs $0.7 \mathrm{ng} / \mathrm{ml}, 0.2-2.9 ; \mathrm{p}=0.05$ ). A multivariate analysis identified PCT and neutrophil count as more reliable predictors than WBC count and CRP ( $\mathrm{p}<0.002,0.04$ vs 0.06 and 0.13 , respectively).

An Italian [24] study performed on 319 children (aged 1 month to 14 years; inpatients), used PCT measurements to guide antibiotic treatment choices. The study design implied that all patients were divided into two subgroups: a PCT-guided therapy group, and an evidence-based therapy group. The former underwent antibiotic therapy based on PCT values $(\geq 0.25 \mathrm{ng} / \mathrm{ml})$. The study found that the PCT group received significantly fewer courses of antibiotics ( $85.8 \%$ vs $100 \%$; $<<0.05$ ), was exposed to antibiotics for less time (5.37 vs 10.96 days; $\mathrm{p}<0.05$ ), and experienced fewer adverse effects to antibiotic treatment (3.9\% vs $25.2 \%$; $<<0.05$ ).

\section{PCT vs other inflammatory markers}

A great effort has been made in order to assess the role of PCT and other inflammatory nonspecific markers, individually and in combination, in CAP management, as well as for detecting etiologic features, in assessing disease prognosis and severity, or in predicting clinical response to antibiotic treatment. Specifically, two research groups $[6,14]$ have tested the diagnostic performance of the above-mentioned predictors with the goal to identify the best one, by assessing the diagnostic characteristics at different thresholds for each one. Since each study has been carried out using different methodological approaches, from the enrollment criteria to laboratory analysis, different cut-offs have been used to assess the diagnostic performance of each marker and to eventually identify the best predictor $[6,10,12,14]$. Toikka [6], in 2000, and Moulin [7], in 2001, chose to evaluate IL-6 in addition to PCT and CRP, in order to assess the etiology of CAP. The results were similar when analyzing the comparison between PCT and IL- 6 diagnostic performances, showing PCT to be more accurate than IL6, while they conflicted when comparing PCT and CRP. In particular, the results of Toikka showed that using PCT $>2 \mathrm{ng} / \mathrm{ml}, \mathrm{CRP}>150 \mathrm{ng} / \mathrm{ml}$ or IL6 $>40$ $\mathrm{pg} / \mathrm{ml}$, the specificity was $>80 \%$ for bacterial pneumonia for all the markers; while the sensitivities with these cut-off values were $50 \%$ for PCT, 31\% for CRP and 34\% for IL-6. As seen before, Toikka found that CRP and PCT values greatly overlapped. However, the results reported by Moulin showed that the concentration PCT, with a threshold of 2 $\mathrm{ng} / \mathrm{ml}$, is more sensitive and specific and has greater positive and negative predictive values than CRP, IL6 or white blood cell count for differentiating bacterial and viral causes of community-acquired pneumonia. The areas under the ROC curves were compared for all of the markers, except WBC count: $\mathrm{p}<0.04$ PCT vs CRP, and $\mathrm{p}<0.003$ for PCT vs IL-6.

The study by Nascimento-Carvalho [10] evaluated the cytokine IFN$\alpha$ in addition to PCT, in relation to its usefulness in differentiating viral from bacterial etiology among children with bacteraemic CAP. The results showed an association between the presence of detectable IFN- $\alpha$ and $\mathrm{PCT}<2 \mathrm{ng} / \mathrm{ml}(\mathrm{p}<0.02)$. The authors concluded that the presence of IFN- $\alpha$ did not add any diagnostic value to PCT in distinguishing between the different etiological groups.

In 2009, Don et al. [14] evaluated the role of the main inflammatory markers, excluding cytokines, by analyzing the association between five different combinations of PCT, CRP, WBC count and ESR and the etiology of pneumonia. The results showed that for all four of the marker values > 50th percentile (CRP: $101 \mathrm{mg} / \mathrm{l}$; WBC: 15.34 cells $\times 10^{9} / \mathrm{l}$; PCT: $1 \mathrm{ng} / \mathrm{ml}$; ESR: $65 \mathrm{~mm} / \mathrm{h}$ ), the likelihood ratio for positive results (LR + ) was 2.7 for separating pneumococcal from viral CAP, and 3.91 for distinguishing atypical from viral CAP. When considering PCT alone at 75th percentile $(18 \mathrm{ng} / \mathrm{ml})$, it had a LR + of 4.14 for distinguishing a pneumococcal from viral etiology, while it was 3.4 in atypical vs viral CAP. The authors concluded that elevated CRP, PCT and WBC had no significant association with the etiology of CAP. However, values of ESR at 50th and 75th percentiles $(60 \mathrm{~mm} / \mathrm{h}, 90 \mathrm{~mm} / \mathrm{h})$ and WBC at 75th percentile ( 22 cells $\times 10 / 1$ ) were significantly associated with the etiology of the infection.

In 2004, Korppi [25] investigated the role of the combination of four inflammatory markers (PCT, CRP, WBC, ESR), and chest radiographic findings for differentiating pneumococcal from viral etiology in 132 children (inpatients from the ages of 1 month to 5 years). Using the 25th, 50th, 75th and 90th percentile values of each marker, the sensitivity, specificity and likelihood ratio for positive results were calculated and, based on the likelihood ratio value, the most accurate combination was selected. The authors concluded that there was no sensitive and specific combination of these markers that could be used in the clinical pediatric practice. Lee et al. [9] obtained similar results when comparing the same markers in children with lobar pneumonia. They found that LR of PCT for diagnosis of lobar pneumonia was maximum at a cut-off value of $2 \mathrm{ng} / \mathrm{ml}$, LR being higher than the CRP level at a cut-off of $6 \mathrm{mg} / \mathrm{L}$ (6.9) and the ESR at a cut-off of $30 \mathrm{~mm} / \mathrm{h}(6.8)$.

With regard to the role of PCT, CRP, ESR and WBC count in assessing disease severity, the study by Don [20] showed that the severity of pneumonia, evaluated by the need for hospital treatment and the presence of an alveolar infiltration on chest radiograph, was associated with a significant elevation of the average serum PCT concentrations compared to outpatients, and that there was a good correlation between PCT and CRP $(r=0.72), W B C(r=0.59)$, and only a moderate correlation with ESR $(r=0.43)$.

Cohen [23] performed an analysis of predictors (PCT, CRP, WBC count and ESR), with regard to the role of inflammatory markers in predicting clinical response to antibiotic treatment, and assessed the usefulness of PCT compared with the three other markers.

\section{Conclusion}

The management of CAP in children still requires useful laboratory tools either for detecting the etiology agent, with the goal to start the correct antibiotic treatment sooner and to reduce antibiotic courses, or for predicting prognosis of the disease. Among the biomarkers, PCT and CRP have been carefully studied and compared, leading to controversial results. In addition, other markers for inflammation have been proposed for CAP diagnosis and management, such as the soluble urokinase plasminogen activator receptor (suPAR), however in this case there is still no definitive data $[26,27,28]$. Overall, the most recent data showed PCT to be the best performance diagnostic marker, especially in terms of detection of a pneumococcal infection [29,30]. As mentioned previously, PCT could lead to early beta lactam therapy, given that it's a predictor of pneumococcal pneumonia.

Unfortunately, the studies reported in this review have some limitations: firstly, sample sizes are small; furthermore, most of them involve children with uncomplicated CAP. Thus, it's still unknown whether there is an advantage in severe and complicated CAP. Currently, the use of CRP in clinical practice has not been replaced by PCT, probably due to its higher cost compared CRP, and to its limited availability. Nevertheless, there is an increase in results confirming the usefulness of PCT for the management pediatric CAP, but further studies are needed for a better understanding its role.

\section{References}

[1] R.H. Snyder, E.S. Nylen, K.L. Becker, Procalcitonin and its component peptides in systemic inflammation: immunochemical characterization, J. Investig. Med. 45 (9) (1997) 552-560.

[2] B. Muller, J.C. White, E.S. Nylen, R.H. Snider, K.L. Becker, J.F. Habener, Ubiquitous expression of the calcitonin gene in multiple tissues in response to sepsis, J. Clin. Endocrinol. Metab. 86 (2001) 396-404

[3] A.M.C. Van Rossum, R.W. Wulkan, A.M. Oudesluys-Murphy, Procalcitonin as an early marker of infection in neonates and children, Lancet Infect. Dis. 4 (10) (2004) 620-630. 
[4] J.E. Clark, D. Hammal, F. Hamptom, D. Spencer, L. Parker, Epidemiology of community-acquired pneumonia in children seen in hospital, Epidemiol. Infect. 135 (2) (2007) 262-269.

[5] M. Tamura, M. Watanabe, A. Nakajima, D. Kurai, H. Ishii, S. Takata, et al., Serial quantification of procalcitonin (PCT) predicts clinical outcome and prognosis in patients with community-acquired pneumonia (CAP), J. Infect. Chemother. 20 (2) (2014) 97-103.

[6] P. Toikka, K. Irjala, T. Juvén, R. Virkki, J. Mertsola, M. Leinonen, et al., Serum procalcitonin, C-reactive protein and interleukin-6 for distinguishing bacterial and viral pneumonia in children, Pediatr. Infect. Dis. J. 19 (7) (2000) 598-602.

[7] F. Moulin, J. Raymond, M. Lorrot, E. Marc, J. Coste, J.L. Iniguez, et al., Procalcitonin in children admitted to hospital with community acquired pneumonia, Arch. Dis. Child. 84 (4) (2001) 332-336.

[8] M. Korppi, M. Don, F. Valent, M. Canciani, The value of clinical features in differentiating between viral, pneumococcal and atypical bacterial pneumonia in children, Acta Paediatr. 97 (7) (2008) 943-947.

[9] J.Y. Lee, S.J. Hwang, J.W. Shim, H.L. Jung, M.S. Park, H.Y. Woo, et al., Clinical significance of serum procalcitonin in patients with community-acquired lobar pneumonia, Korean J. Lab. Med. 30 (4) (2010) 406-413.

[10] C.M. Nascimento-Carvalho, M.R. Cardoso, A. Barral, C.A. Araújo-Neto, S. Guerin, A. Saukkoriipi, et al., Procalcitonin is useful in identifying bacteremia among children with pneumonia, Scand. J. Infect. Dis. 42 (9) (2010) 644-649.

[11] A. Galetto-Lacour, G. Alcoba, K.M. Posfay-Barbe, M. Cevey-Macherel, M. Gehri, M.M. Ochs, et al., Elevated inflammatory markers combined with positive pneumococcal urinary antigen are a good predictor of pneumococcal community-acquired pneumonia in children, Pediatr. Infect. Dis. J. 32 (11) (2013) 1175-1179.

[12] M. Korppi, S. Remes, Serum procalcitonin in pneumococcal pneumonia in children, Eur. Respir. J. 17 (4) (2001) 623-627.

[13] M. Korppi, S. Remes, T. Heiskanen-Kosma, Serum procalcitonin concentrations in bacterial pneumonia in children: a negative result in primary healthcare settings, Pediatr. Pulmonol. 35 (2003) 56-61.

[14] M. Don, F. Valent, M. Korppi, M. Canciani, Differentiation of bacterial and viral community-acquired pneumonia in children, Pediatr. Int. 51 (1) (2009) 91-96.

[15] P. Berg, B. Lindhardt, The role of procalcitonin in adult patients with community acquired pneumonia-a systematic review, Dan. Med. J. 59 (3) (2012) a4357.

[16] J. Hedlund, L. Hansson, Procalcitonin and $C$ reactive protein levels in community acquired pneumonia: correlation with etiology and prognosis, Infection 28 (2000) 68-73.

[17] Y.Y. Han, L.A. Doughty, D. Kofos, H. Sasser, J.A. Carcillo, Procalcitonin is persistently increased among children with poor outcome from bacterial sepsis, Pediatr. Crit. Care Med. 4 (2003) 21-25.

[18] D.C. Van der Kaay, E.D. De Kleijn, Y.B. De Rijke, W.C. Hop, R. De Groot, J.A. Hazelzet, Procalcitonin as a prognostic marker in meningococcal disease, Intensive Care Med. 28 (2002) 1606-1612.
[19] C. Prat, J. Dominguez, C. Rodrigo, M. Giménez, M. Azuara, O. Jiménez, et al., Elevated serum procalcitonin values correlate with renal scarring in children with urinary tract infections, Pediatr. Infect. Dis. J. 22 (2003) 438-442.

[20] M. Don, F. Valent, M. Korppi, E. Falleti, A. De Candia, L. Fasoli, et al., Efficacy of serum procalcitonin in evaluating severity of community acquired pneumonia in children, Scand. J. Infect. Dis. 39 (2007) 129-137.

[21] M. Harris, J. Clark, N. Coote, P. Fletcher, A. Harnden, M. McKean, et al., British Toracic Society Guidelines for the management of community acquired pneumonia in children: update 2011, Thorax 66 (2011) ii1-ii23.

[22] J.S. Bradley, C.L. Byington, S.S. Shah, B. Alverson, E.R. Carter, C. Harrison, et al., The management of community acquired pneumonia in infants and children older than three months of age. Clinical practice guidelines by the pediatric infectious diseases society and the infectious diseases society of America, Clin. Infect. Dis. 53 (2011) e25-e76.

[23] J. Cohen, A. Leis, T. Lecarpentier, J. Raymond, D. Gendrel, M. Chalumeau, Procalcitonin predicts response to beta lactam treatment in hospitalized children with community acquired pneumonia, PLoS One 7 (5) (2012), e36927.

[24] S. Esposito, C. Tagliabue, I. Picciolli, M. Semino, C. Sabatini, S. Consolo, et al., Procalcitonin measurements for guiding antibiotic treatment in paediatric pneumonia, Respir. Med. 105 (12) (2011) 1939-1945.

[25] M. Korppi, Non-specific host response markers in the differentiation between pneumococcal and viral pneumonia: what is the most accurate Combination? Pediatr. Int. 46 (5) (2004) 545-550.

[26] A. Wrotek, T. Jackowska, K. Pawlik, Soluble urokinase plasminogen activator receptor: an indicator of pneumonia severity in children, Adv. Exp. Med. Biol. 835 (2015) $1-7$.

[27] Y. Backes, K.F. van der Sluijs, D.P. Mackie, et al., Usefulness of suPAR as a biological marker in patients with systemic inflammation or infection: a systematic review, Intensive Care Med. 38 (9) (2012) 1418-1428.

[28] R.B. Raggam, J. Wagner, F. Prüller, et al., Soluble urokinase plasminogen activator receptor predicts mortality in patients with systemic inflammatory response syndrome, J. Intern. Med. 276 (6) (2014) 651-658.

[29] C.H. Yo, P.S. Hsieh, S.H. Lee, et al., Comparison of the test characteristics of procalcitonin to C-reactive protein and leukocytosis for the detection of serious bacterial infections in children presenting with fever without source: a systematic review and meta-analysis, Ann. Emerg. Med. 60 (5) (2012) 591-600.

[30] J.M. Pereira, A. Teixeira-Pinto, C. Basílio, C. Sousa-Dias, P. Mergulhão, J.A. Paiva, Can we predict pneumococcal bacteremia in patients with severe community-acquired pneumonia? J. Crit. Care 28 (6) (2013) 970-974. 